This document was prepared in conjunction with work accomplished under Contract No. DE-AC09-96SR18500 with the U.S. Department of Energy.

This work was prepared under an agreement with and funded by the U.S. Government. Neither the U. S. Government or its employees, nor any of its contractors, subcontractors or their employees, makes any express or implied: 1 . warranty or assumes any legal liability for the accuracy, completeness, or for the use or results of such use of any information, product, or process disclosed; or 2 . representation that such use or results of such use would not infringe privately owned rights; or 3 . endorsement or recommendation of any specifically identified commercial product, process, or service. Any views and opinions of authors expressed in this work do not necessarily state or reflect those of the United States Government, or its contractors, or subcontractors. 


\title{
Multi-Point Radiation Monitor
}

\author{
K. J. Hofstetter, D. M. Beals, D. M. Odell, R. F. Eakle, R. K. Huffman, L. J. Harpring \\ Washington Savannah River Company \\ Savannah River National Laboratory \\ Aiken, SC 29808
}

\begin{abstract}
A unique radiation monitor has been developed for performing wide-area field surveys for radiation sources. This device integrates the real-time output of multiple radiation detectors into a hand-held personal computer (e.g., a PDA) containing an intuitive graphical user interface. An independent hardware module supplies high voltage to the detectors and contains a rapid sampling system for transferring the detector count rates through an interface to the PDA. The imbedded firmware can be changed for various applications using a programmable memory card. As presently configured, the instrument contains a series of Geiger-Mueller (GM) tubes in a flexible detector string.

This linear array of multiple sensors can be used by US Coast Guard and Customs container inspection personnel to measure radiation intensity in stacks of transport containers where physical access is impeded.
\end{abstract}

\section{Introduction}

Various federal, state and local agencies are charged with intercepting illicit nuclear materials before they enter the country. One potential method for smuggling nuclear materials into the US would be to hide it among containerized cargo inside a SeaLand container. Nearly 9 million containers loaded with commercial cargo enter the US yearly through official maritime points of entry. The containers are tightly stacked on-board container ships, unloaded at port terminals and then temporarily stored dock-side waiting intermodal transport. Dockside inspection of every container would produce unacceptable delays in the flow of commerce.

An instrument was developed and tested that can be used by field inspection personnel at the nation's seaports. The instrument includes ruggedized radiation detectors assembled into a flexible linear array that can be slipped between stacks of containers to detect the presence of radiation sources and determine their relative location. A prototype system was developed consisting of field hardened radiation detectors and an operator interface capable of measuring the radiation field emanating from an individual SeaLand container when stacked on board ships or at port storage yards without moving the containers. A series of individual radiation sensors placed along a length of flexible cable communicating via a unique 1-wire communications network to a remote operator acts as a "RadRope". The RadRope extends the operators field of inspection to measure the radiation field at remote locations and identify its approximate location.

\section{System Design}


A series of inexpensive yet rugged Geiger-Mueller (GM) tubes were networked using a novel 1-wire communications technology to sense radiation along the length of the sensor array. Each detector was independently monitored and uniquely identified. The combination of multiple radiation sensors and communication technology established a networked sensor array system. The multiple linear array of GM tubes, costing only $\$ 150$ each, could easily be field hardened for this application. A programmable PDA was used as a simple graphical user interface to control the array of detectors and perform simple calculations of backgrounds and alarm levels.

The design of this multi-detector array uses a unique sensor node design that minimizes complexity, manufacturing and repair costs while maximizing reliability. The detailed design is scalable to permit manufacturing of systems with varying number and types of sensors. The initial prototype employed 8 sensors spaced approximately 3 feet apart encased in a flexible, strong outer sheath. Each detector node contained a GM tube, resistor-capacitor charge circuit, and 1-wire network analog to digital converter (ADC). A laptop computer was used initially for hardware testing and software development. Visual Basic 6 software controlled the Dallas Semiconductor's one wire communications adaptor specifically using the DS2450 quad ADCs. The laptop was also used to develop the custom graphical user interface.

The data acquisition software queries the one wire network to find all the analog to digital converters, configures each one, and repeatedly reads the converted values. The time to read all eight detectors and the read/write memory cycle time limited the polling time to approximately once every 3 seconds in the initial design. The graphical user interface (GUI) was developed in Visual Basic for porting into the embedded Visual Basic in a Viewsonic Pocket PC. The existing serial communications port on the Pocket $\mathrm{PC}$ was then used to communicate with the detector string. An example of the GUI output is shown in the following figure.

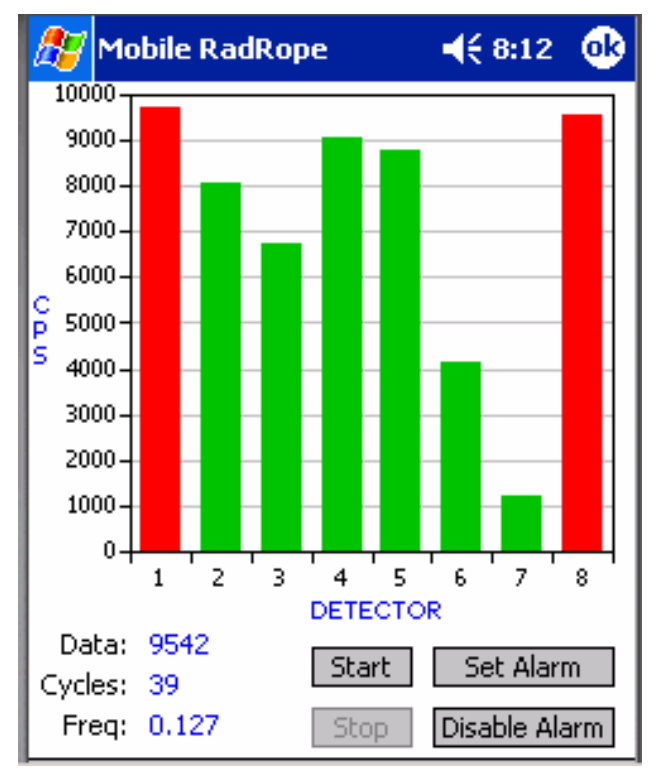

Figure 1. Prototype Multi-detector Array Output 
The RadRope prototype includes a power source, node interface electronics, and a PDA computational unit to process and display the information. The commercially available PDA contains SRNL proprietary software for hardware control and user interface. The PDA is encased in a waterproof, shock proof case to withstand the rigors of field use. The entire unit is powered by a Lithium-polymer battery. A 500 volt DC bussed power for the GM tubes is supplied along with a 5 volt DC bus to power the ADC, the 1-wire signal bus for communications and a common ground. The ADCs and communications interface are mounted on flexible circuit boards for each detector. The detectors, electronic circuit cards and wiring are encased in heavy duty, flexible fabric as part of the field hardening design. The GM tube output charges the RC network to a voltage proportional to the radiation field. The ADC is polled by the user interface module and the measured voltage for each detector interpreted by the processor as the corresponding radiation level for each detector.

The data control unit is shown in Figure 2. Control of the instrument is accomplished by four buttons on the PDA. Two buttons on the left are used to Start and Stop data acquisition, while the two buttons on the right are used to set the alarm levels for operator alert. When any of the detectors reach the alarm level count rates, the bar display for that individual detector changes from green to red (See Figure 1). An audible alarm is also generated. The audible alarm can be disabled by the operator during setup. Following an alarm, the operator must acknowledge the alarm by pushing the "Toggle Alarm" pushbutton. The LEMO shunt connector shown on the lower right protruding from the protective case must be removed for offline battery charging. 


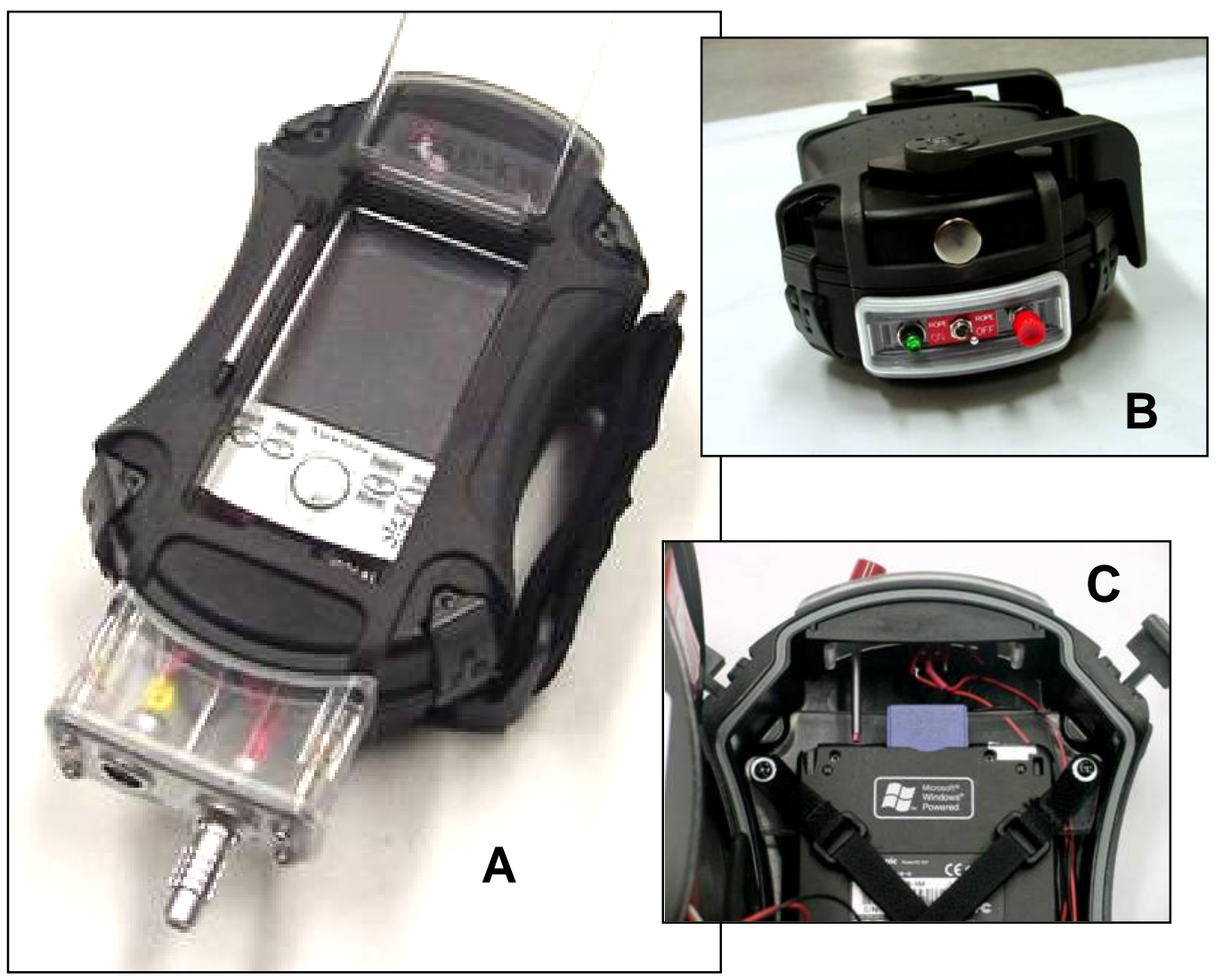

Figure 2. The Data Control Unit and Graphical User Interface

Setting up and operating the instrument is accomplished with a simple 5 step procedure.

1. Plug the string of radiation detectors into the LEMO connection on the bottom of the control unit.

2. Turn on the voltage to the detectors by flipping the toggle switch to the ON position. (See Figure 2B.)

3. Turn on the Pocket PC by quickly pushing and releasing the red button on the top of the control unit. (See Figure 2B.)

4. Select Mobile from the Pocket PC menu and wait for program loading.

5. Press Start to begin data acquisition. The audible alarm is enabled automatically. To disable, press the "Toggle Alarm" button.

To charge the internal batteries, unplug the detector string and the LEMO shunt and plug in the battery charger. The internal batteries can be charged from a standard $110 \mathrm{VAC}$ source or from a 12 VDC battery using the cigarette lighter adapter. The operating lifetime of the internal batteries is about 4 hours when fully charged. A belt-worn supplementary battery is available for extending the operating life of the unit.

Porting of information from the host computer to the PDA during development was accomplished using a standard solid state flash memory card. See Figure 2C. 


\section{Experimental}

To test the initial 1-wire communications concept for radiation count rate monitoring, small GM tubes were used for bench scale testing. These commercially available GM tubes were nominally 0.5 " in diameter and 2 " long. The manufacturer's quoted sensitivity for these tubes was $\sim 1.5$ counts per second (cps) for a ${ }^{60}$ Co source of $1 \mathrm{mR} / \mathrm{hr}$ dose rate. This count rate response was adequate for proof of principle testing on the bench using radioactive point sources. Four of these detectors were wired in series during breadboard testing to successfully demonstrate the networked sensor array approach. As a result of these breadboard tests, larger GM tubes were ordered for the field prototype to provide adequate detection sensitivity for meaningful field measurements. For the initial prototype, eight seven inch long by $1 / 2$ inch diameter GM tubes were ordered from LND Corporation with sensitivities of $60 \mathrm{cps} / \mathrm{mR} / \mathrm{hr}$ generated by ${ }^{60} \mathrm{Co}$.

The first series of tests of the prototype was performed using point sources in the laboratory. The GM tubes were not energy compensated due to their relatively thick walls; a study of their energy response function was performed to determine their response to various radionuclides. The results of these tests are summarized in the following Figure. A crude correlation of count rate as a function of maximum gamma ray energy can be inferred from this figure as was anticipated. Since no spectroscopic information can be obtained from the GM tubes, these data showed adequate response for detecting most gamma emitting radionuclides for this application.

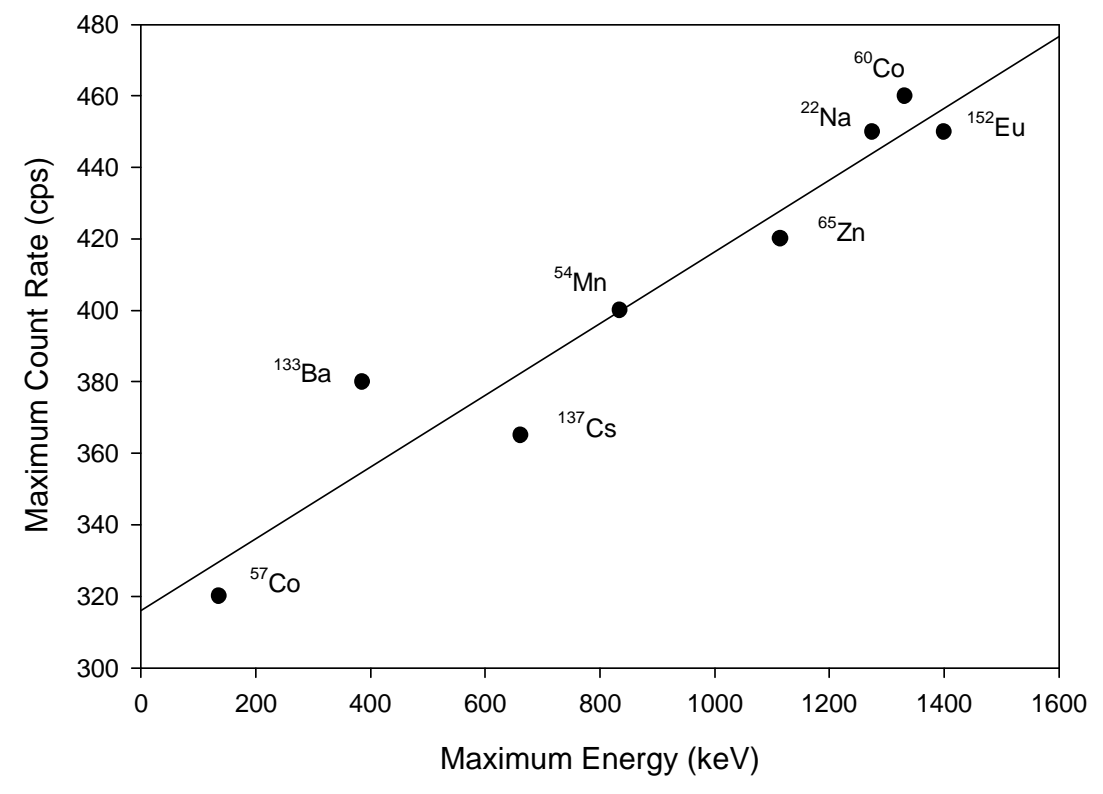

Figure 3. Response to Various Radionuclides (average of all detectors) 
Repeated tests in the laboratory environment showed the prototype with eight GM tubes yielded stable background count rates from each detector and consistent count rates when exposed to radioactive sources. It was noted however, that the count rate did not reach an equilibrium value before several polling cycles had been conducted when exposed to a radioactive source. An improved system design was undertaken to reduce the polling time and improve the rapid response of the detectors to change in dose rate field. The instrument response time was determined to be a combination of the detector integration time constant and the speed of data transfer from the detector to the PDA. Several design improvements were implemented to improve this parameter. The time constant of the detector circuit was modified to shorten the integration time. Higher voltage GM tubes were used to increase sensitivity. Embedded controllers were added to interface between the PDA and the detectors. The embedded controllers handle communication with the individual detectors, streamlining the communication with the PDA which proved to be a bottleneck in the system.

\section{System Improvements}

Another embedded controller is being added to the detector PC board, allowing the system to create a faster local area network which will improve the communication rate from 2400 baud to $2 \mathrm{M}$ bps. This will also allow implementation of a pulse counting algorithm to further increase the response and sensitivity of the instrument. The hardware design for these changes has been completed and the software implementation is under development.

The present version of the device utilizes a chain of eight detectors hardwired together. This chain of eight detectors was then interfaced to the PDA. When the software was initiated a source was used to identify the location of each detector in the chain. In the latest version, any number of detectors, from one to ten, can be interconnected. Each detector segment has a connector on each end and a terminator is placed on the end of the last detector segment to complete the chain. When the software is initiated the PDA automatically identifies each detector segment and its place in the chain. Sourcing the detectors to find their respective location is not required.

\section{Applications Tested}

The prototype instrument has been extensively tested in the field at multiple ports both on board ships docked in port and in various laydown yard locations. The first series of field tests were performed in Charleston, SC at several of their large terminals. The RadRope was taken on board a foreign-flagged containership docked at the Wando-Welch terminal under escort by a Coast Guard inspector from the Charleston Marine Safety Office. The ship's Captain permitted the researchers to climb on top of a stack of SeaLand containers loaded on the weather deck and survey the containers with the RadRope. Ladders were used to gain access. The detector string was then lowered between the stack of containers and count rates measured as the stack was traversed. In this case, the count rates observed were slightly lower than the count rates obtained with the RadRope lying on top of the container stack. Shielding from the containers and contents were probably responsible for 
the slight decrease in average count rate. The average count rate on board ship was considerably less than at dock-side. The container ship was in the process of off-loading. This medium sized container ship had containers stacked 6 high on the weather deck which makes it difficult to reach the top of the stack from a safety perspective, especially at sea. See Figure 4. It was observed that the containers stored below the weather deck were in covered holds which were easily accessible when the hatch cover was removed. A typical large container ship has containers stacked 7 high below deck. This ease of access would permit an inspector to quickly scan the containers below deck with the device after the hatch cover was removed before unloading. According to the Coast Guard inspector, this could easily and safely be accomplished as a rapid means of performing a radiation scan of the containers prior to unloading.

The prototype was also tested on board a large water transport ship used for transporting automobiles and other rolling stock, often called roll-on roll-off (RORO) carrier. The RadRope was taken onboard the docked RORO ship at the Union Pier terminal in Charleston under USCG escort. See Figure 5. The ship in port was in the process of offloading Mercedes Benz automobiles and being made ready to load BMW autos for transport to Europe. Many other vehicles were shipped on this RORO including mobile homes, luxury yachts on trailers and privately owned special vehicles. There were many opportunities to use the RadRope to scan these vehicles while on-board ship and also to set up a portable portal monitor using the instrument to screen the vehicles as they were leaving the ship. Many of the miscellaneous vehicles were scanned quickly by taking advantage of the multi-detector capability.

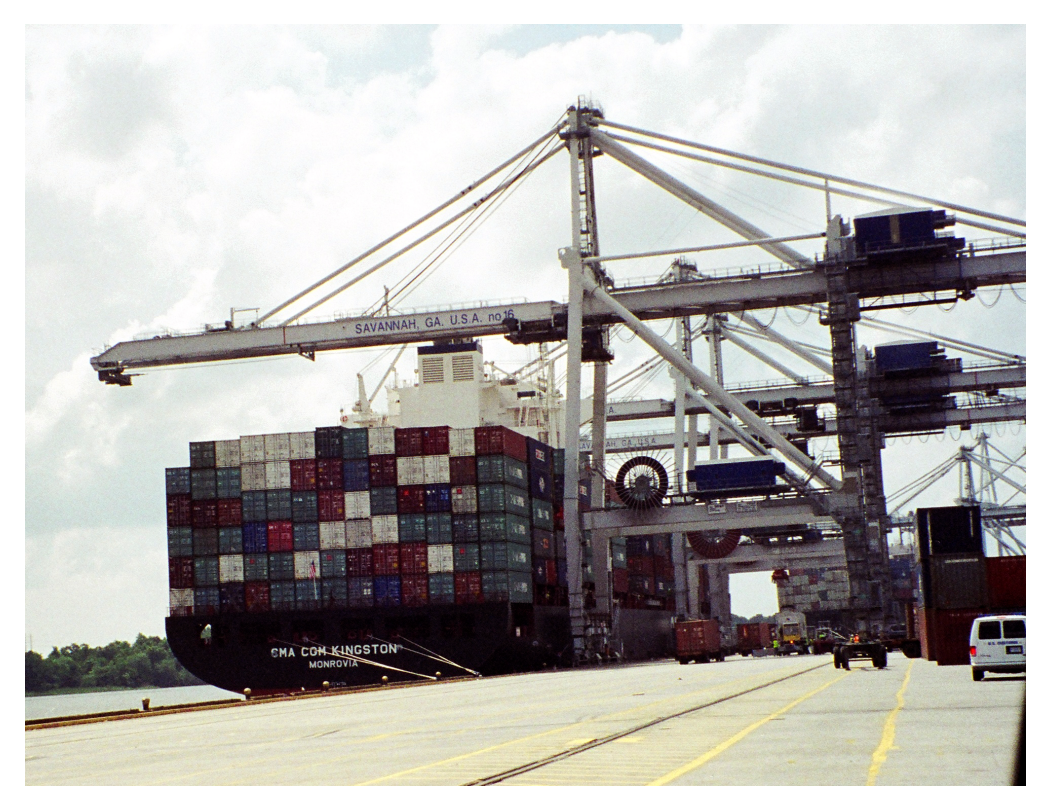

Figure 4. Container Ship Docked at Wando-Welch Terminal in Charleston, SC 


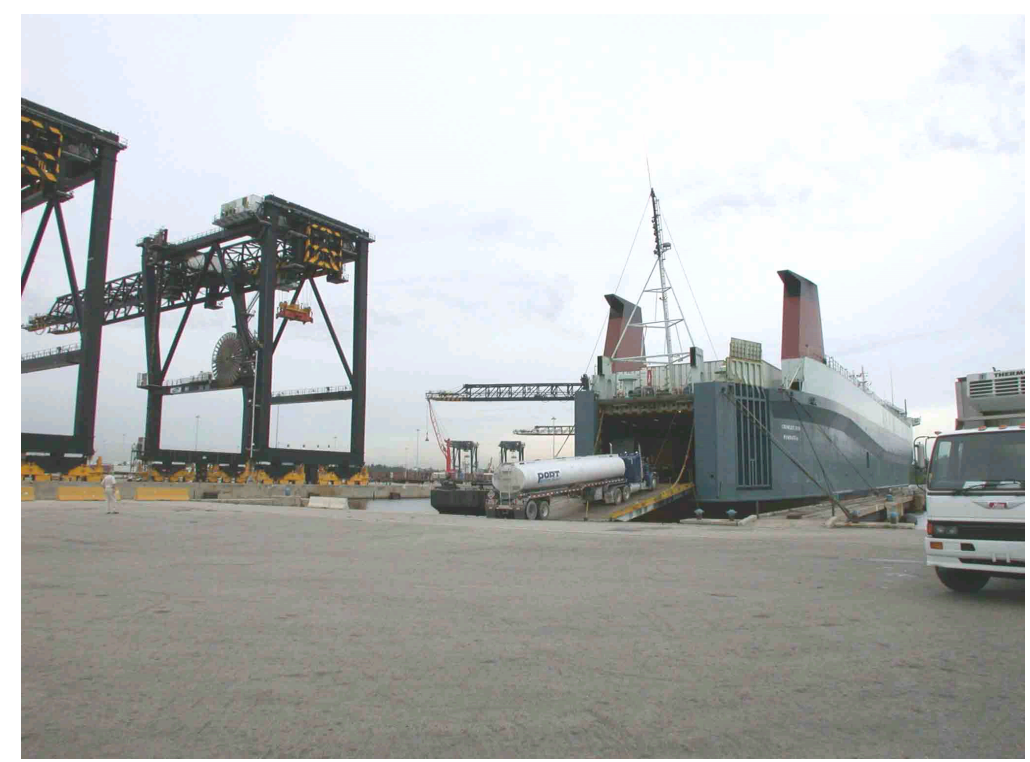

Figure 5. RORO Transport Ship

There were many opportunities to test the prototype at dockside where much of the cargo is temporarily stored in preparation for shipment. The cargo containers are typically stacked in a close-packed configuration as shown in Figure 6. At this terminal in Charleston, the maximum stack is 4 containers high. Often these containers stacks are too close to use the RadRope as indicated in the insert in Figure 8. Under those conditions, the RadRope can be attached to a pole and used to vertically scan the outside row of containers and also slide under the lowest container for a horizontal scan of the bottom container contents. With the RadRope attached to the pole, the top of a sea-land container loaded on a truck for final transport could be performed. This operation was demonstrated to the Coast Guard and Customs and Border Protection agents.

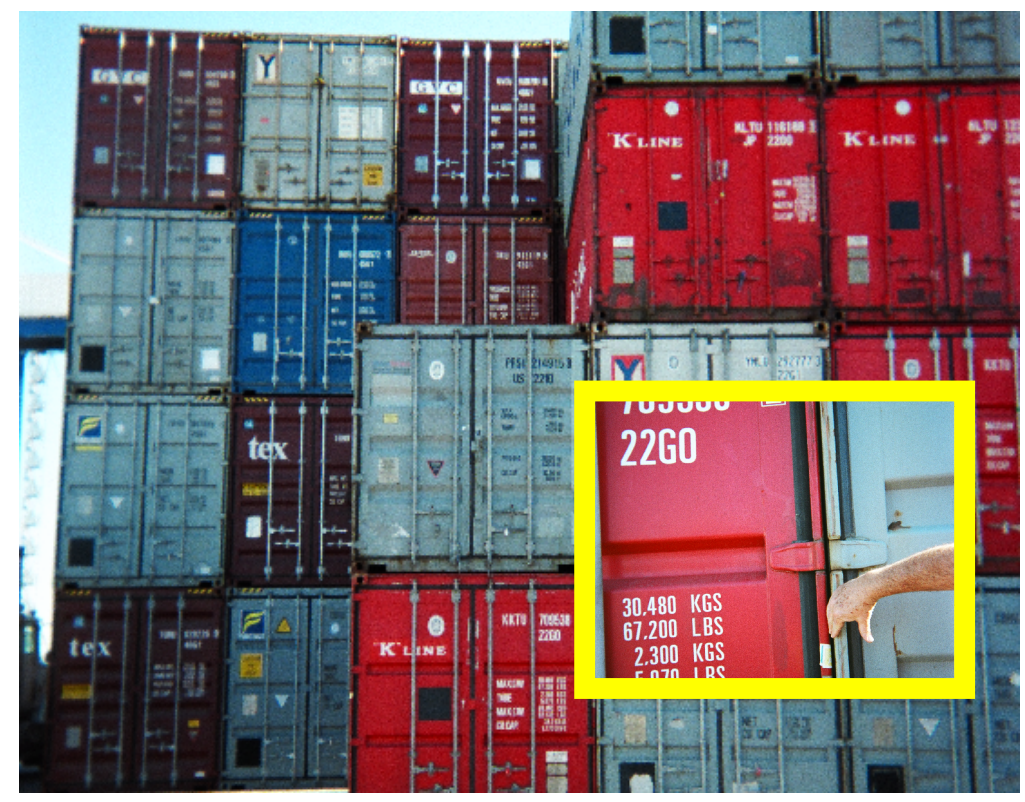


Figure 6. Stack of Cargo Containers in Charleston

Similar demonstrations and field testing of the technology was performed for Coast Guard and CBP agents in Seattle. Valuable feedback on how the instrument might fit into their respective inspection regimes was obtained. While in Seattle, the RadRope was taken onboard a commercial fishing and processing vessel for testing. There were many possible applications for a portable, flexible, extended radiation detector for inspecting a vessel containing complex machinery for illicit radioactive material.

\section{Conclusions}

A simple, inexpensive instrument for measuring radiation fields in otherwise inaccessible locations has been developed. The RadRope consists of a series of radiation detectors inside a flexible sheath and connected to a data readout device to alert the operator to unexpected radiation fields at a remote location. The instrument is designed for use in a maritime environment and will assist inspection personnel in detecting potential illicit radioactive materials or rad/nuc weapons of mass destruction. 